\title{
Acquisition and transfer of control by an ambiguous cue
}

\author{
PETER C. HOLLAND and CHRISTINE E. REEVE \\ Duke University, Durham, North Carolina
}

\begin{abstract}
In three experiments, the specificity of action of occasion setters was examined, using a discretetrial leverpress procedure. Rats' acquisition of an $X A+, A-, X B-, B+$ discrimination, in which a single feature cue $(X)$ signaled the reinforcement of responding in the presence of one target cue $(A)$ and the nonreinforcement of responding during another $\operatorname{target}(B)$, was no more difficult than acquisition of $X A+, A-, X-, B+$ or $X+, A-, X B-, B+$ discriminations, in which $X$ signaled only one target-response-reinforcement contingency. In transfer tests, $X$ did not modulate responding controlled by other cues that were untrained or consistently reinforced, either when the elements of the compounds were presented serially or when they were presented simultaneously in training and testing. However, after serial, but not after simultaneous, compound training, $X$ facilitated responding controlled by a cue that was trained and then extinguished. Implications for the nature of occasion setting and configural learning are discussed.
\end{abstract}

The results of many recent studies (e.g., Holland, 1989a, 1989 d) suggest that the temporal arrangement of elements within a compound stimulus $(X A)$ can affect the way rats solve Pavlovian appetitive feature-positive $(X A+, A-)$ and feature-negative $(A+, X A-)$ discriminations. Holland (1983) claimed that when $X$ and $A$ are presented simultaneously, excitatory (in feature-positive discriminations) or inhibitory (in feature-negative discriminations) associations are usually formed between $\boldsymbol{X}$ and the unconditioned stimulus (US). However, if the onset of $X$ precedes the onset of $A, X$ acquires the ability to modulate the action of the association between $A$ and the US, setting the occasion (Moore, Newman, \& Glasgow, 1969; Skinner, 1938) for either responding or nonresponding to $A$. Considerable experimentation showed that this occasion setting or modulation differs from simple excitation and inhibition in many ways. Indeed, there is abundant evidence that simple excitatory or inhibitory and modulatory powers of Pavlovian conditioned stimuli (CSs) are largely independent (e.g., Holland, 1983; Rescorla, 1985).

The experiments reported here explored the locus of occasion setters' modulation of $\boldsymbol{A}$-US associations. Rescorla (1985) suggested that they acted very generally, by either raising or lowering the activation threshold of a representation of the US. In an $X A+, A$ - discrimination, for example, $A$ 's associative strength is insufficient to activate the US representation, unless the presentation of $X$ has lowered the activation threshold of that representation. Conversely, Holland (1983) proposed that $X$ acted on a specific CS-US association, in a hierarchical fashion. In

This research was supportod by a grant from the National Science Foundation. Christine E. Reeve is now at the State University of New York, Stonybrook. Correspondence should be directed to Peter C. Holland, Psychology Department, Duke University, Durham, NC 27706. a feature-positive discrimination, the particular $A$-US association is ineffective in activating the US representation, unless it is empowered by $X$.

In these experiments, we examined the specificity of action of occasion setters by studying rats' acquisition of an $X A+, A-, X B-, B+$ discrimination, in which a single occasion setter $(X)$ signaled the reinforcement of one target cue $(A)$ and the nonreinforcement of another cue $(B)$. If, as Rescorla suggested, modulatory stimuli act by altering the activation threshold of the US, then this discrimination could not be solved by the use of modulatory processes alone: some additional mechanism would be required to ensure that $X$ lowered the threshold for $A$ but raised it for $B$. However, if modulators acted on specific target-US associations, it is easy to imagine that $\boldsymbol{X}$ could simultaneously enable the $A$-US association and disable the $B$-US association.

The experiments reported here used discrete-trial operant, rather than Pavlovian, conditioning procedures. In a series of experiments in which these operant procedures were used to study learning in serial and simultaneous feature-positive $(X A+, A-)$ and feature-negative $(X A-, A+)$ discrimination paradigms, Holland (in press) found substantial similarities with his previously reported data from Pavlovian procedures (e.g., Holland, 1989b). The current experiments extend that work.

\section{EXPERIMENT 1}

Experiments 1 and 2 compared the acquisition and transfer of discriminative performance in the ambiguous training procedure $(X A+, A-, X B-, B+)$ with that found with positive-patterning $(X A+, A-, X-)$ and negativepatterning $(X B-, B+, X+)$ procedures. The latter procedures arrange the same simple relations between $X$ and reinforcement and/or nonreinforcement as the ambiguous 
discrimination procedure, but only one conditional relation. If $X$ independently modulates $A$-US and $B$-US associations, then the ambiguous discrimination should be no more difficult than the separate patterning discriminations. However, to the extent that $X$ 's modulatory power involves only the US, the ambiguous discrimination should be more difficult than the patterned discriminations. In Experiment 1, to encourage the use of occasion-setting strategies, the elements of the compound cues were presented serially; in Experiment 2, they were presented simultaneously. The physical and temporal parameters of the cues were those which Holland (e.g., 1989a, 1989d) found to generate different kinds of learning in Pavlovian serial and simultaneous feature-positive and featurenegative discrimination procedures.

\section{Method}

Subjects and Apparatus. The subjects were 15 male and $6 \mathrm{fe}-$ male albino rats bred in the Psychology Department of Duke University. They were about 240 days old at the beginning of the experiment, and had not been involved in previous research. The rats were maintained at $80 \%$ of their ad-lib body weights throughout the experiment by limiting their access to food. Water was available at all times in their individual home cages.

There were eight identical experimental chambers, each $22.9 \times 20.3 \times 20.3 \mathrm{~cm}$ (but only seven chambers were used in Experiment 1). The front and back walls of each chamber were aluminum; the side walls and top were clear acrylic. A food cup was recessed behind a $5 \times 5 \mathrm{~cm}$ opening in the front wall; the bottom of the opening was $2 \mathrm{~cm}$ from the floor, and its center was $2 \mathrm{~cm}$ to the right of the center of the front wall. A $6-\mathrm{W}$ jeweled lamp (panel light) was centered on the front wall, $4 \mathrm{~cm}$ above the top of the food-cup opening. Four centimeters left of the food-cup opening was a $2 \times 2 \mathrm{~cm}$ lever, mounted $3 \mathrm{~cm}$ above the floor. During the first four sessions of leverpress training, the lever was enlarged by a $3.3 \times 1.5 \mathrm{~cm}$ metal paper clamp that was clipped over it. The floor of the chamber was composed of $0.48-\mathrm{cm}$ stainless steel rods spaced $1.9 \mathrm{~cm}$ apart. Each of the chambers was enclosed in a sound- attenuating shell. A normally off 6-W houselight was mounted on one wall of the shell, $10 \mathrm{~cm}$ above the top of the chamber and $2 \mathrm{~cm}$ in front of and $10 \mathrm{~cm}$ to the left of the front wall of the chamber; $10 \mathrm{~cm}$ below the houselight was a speaker for delivering the auditory CSs. Thus, from a position facing the food cup, the houselight was to the left and above the front panel of the chamber and the panel light was in the center, near the top of that wall panel. Finally, each shell was enclosed in another sound-attenuating box. Constant background noise $(62.5 \mathrm{~dB})$ was provided by a ventilating fan on each box.

Procedure. First, the rats were trained to press the lever. In the first, 50-min, session, the rats received $200.3-\mathrm{ml}$ deliveries of $0.2 \mathrm{M}$ sucrose (the reinforcer used throughout these experiments) on a variable-time (VT) 1-min schedule. Leverpresses were reinforced during that period and during the remaining $30 \mathrm{~min}$ of the session. In the second session, each rat was allowed to remain in its chamber until it had made about 50 leverpresses. Leverpresses were reinforced, but there were no response-independent sucrose presentations in this session. For the next four sessions, the objective was to place leverpressing under the control of a clicker in order to both strengthen the leverpress tendency and minimize intertrial (ITI) leverpressing. Each of the first two of these sessions was $50 \mathrm{~min}$ long, and included 2560 -sec presentations of a clicker $(8 \mathrm{~Hz}, 64 \mathrm{~dB})$ during which each leverpress was reinforced. Leverpresses in the absence of an explicit cue were not reinforced in these sessions or in the remainder of the experiment. Each of the next two sessions was $90 \mathrm{~min}$ long, and included 4560 -sec clicker presentations during which leverpressing was reinforced.

Next, the rats were divided into three groups of 7 rats each, and discrimination training was begun. All rats received four kinds of trials in each of the 4090 -min conditioning sessions. Table 1 provides an outline of the procedures of Experiment 1. The rats in Group AMB received training in which a single visual cue $(X)$ indicated the availability of reinforcement during $A$ and the nonavailability of reinforcement during $B$. They received the following trials: (1) $X \rightarrow 0 \rightarrow A+$, which consisted of a 5-sec visual cue $(X)$ followed, after a 5 -sec empty interval, by a $5-\sec 1500-\mathrm{Hz}, 74 \mathrm{~dB}$ tone (for 4 of the subjects, the visual cue was the steady illumination of the houselight, and for the other 3 , it was the intermittent $[3 \mathrm{~Hz}]$ illumination of the panel light; leverpresses during the 5-sec tone were reinforced). (2) $A$-, which were 5-sec presentations of the tone

Table 1

Outline of Procedures of Experiments 1 and 2

\begin{tabular}{|c|c|c|c|c|c|c|c|}
\hline & \multicolumn{7}{|c|}{ Experiment 1} \\
\hline & Discrimination & Test 1 & Test 2 & $C+$ & Test 3 & $C-$ & Test 4 \\
\hline & $\begin{array}{l}X \rightarrow 0 \rightarrow A+, A- \\
X \rightarrow 0 \rightarrow B-, B+\end{array}$ & $\begin{array}{l}X \rightarrow 0 \rightarrow A- \\
X-, A-\end{array}$ & $\begin{array}{l}X \rightarrow 0 \rightarrow B- \\
X-, B-\end{array}$ & $C+$ & $\begin{array}{l}X \rightarrow 0 \rightarrow C- \\
X-, C+\end{array}$ & $C-$ & $\begin{array}{l}X \rightarrow 0 \rightarrow C- \\
X-, C-\end{array}$ \\
\hline Group PP & $\begin{array}{l}X \rightarrow 0 \rightarrow A+, A- \\
X \rightarrow 0 \rightarrow 0-, B+\end{array}$ & $"$ & $"$ & " & 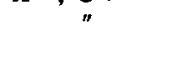 & $"$ & 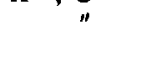 \\
\hline Group NP & $\begin{array}{l}X \rightarrow 0 \rightarrow 0+, A- \\
X \rightarrow 0 \rightarrow B-, B+\end{array}$ & " & $"$ & $"$ & $"$ & $"$ & $"$ \\
\hline
\end{tabular}

Experiment 2

\begin{tabular}{|c|c|c|c|c|c|c|}
\hline & Discrimination & Test 1 & $C+$ & Test 2 & $C-$ & Test 3 \\
\hline & $\begin{array}{l}X A+, A- \\
X B-, B+\end{array}$ & $\begin{array}{l}X A-, A- \\
X B-, B-, X-\end{array}$ & $C+$ & $\begin{array}{l}X C-, C+ \\
X B-, B+, X-\end{array}$ & $C-$ & $\begin{array}{l}X C-, C- \\
X A-, A-, X-\end{array}$ \\
\hline Group PP & $\begin{array}{l}X A+, A- \\
X-, B+\end{array}$ & $"$ & $"$ & & $"$ & 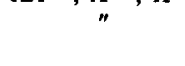 \\
\hline Group NP & $\begin{array}{l}X+, A- \\
X B-, B+\end{array}$ & $"$ & $"$ & " & $"$ & $"$ \\
\hline
\end{tabular}

Note- + signifies availability of sucrose reinforcement, - signifies no reinforcement, $A$ was a tone, $B$ was a noise, $C$ was a clicker, and $X$ was a houselight for half the rats in each group and a panel light for the other half. In Experiment 1,0 signifies a 5-sec empty interval. All stimuli were $5 \mathrm{sec}$ in duration in Experiment 1 and $15 \mathrm{sec}$ in Experiment 2 . In all test sessions of Experiment 1, there were other trial types in addition to those listed here (see text); data are presented only from the trial types included here. 
during which no reinforcement was available. (3) $B+$, in which leverpresses were reinforced during 5-sec presentations of a 73-dB white noise. (4) $X \rightarrow 0 \rightarrow B-$, which consisted of a 5-sec presentation of the visual cue followed, after a 5-sec empty interval, by the noise, during which sucrose was unavailable.

The rats in Group PP received positive-patterning training, in which reinforcement was available during $A$ on $X \rightarrow 0 \rightarrow A+$ trials but not during either $X$ or $A$ alone. They received the same kinds of trials as Group AMB, except that $X \rightarrow 0 \rightarrow B$ - trials were replaced by $X \rightarrow 0 \rightarrow 0-$ trials, in which the 5-sec visual cue (houselight for 4 of the rats and panel light for the others) was presented by itself; reinforcement was unavailable. Finally, the rats in Group NP received negative-patterning training, in which reinforcement was available during presentations of both $B$ and $X$ alone but not during $B$ on $X \rightarrow 0 \rightarrow B$ compound presentations. They received the same kinds of trials as Group AMB, except that $X \rightarrow 0 \rightarrow A+$ trials were replaced by $X \rightarrow 0 \rightarrow 0+$ trials, which comprised a 5 -sec presentation of $X$ (houselight for 4 of the rats and panel light for the others) followed by a 5-sec nonreinforced empty interval and then another 5-sec empty interval during which responding was reinforced.

Each group received identical trial sequences, which were generated randomly (without constraint) for each session. ITIs were randomized daily, with the constraint that the range of intervals was from 0.5 to 2.0 times the mean ITI. In the first 4 discrimination training sessions, there were 10 presentations of each trial type (mean ITI = $135 \mathrm{sec}$ ); in the next 4 sessions, there were 15 of each trial type (mean ITI = $90 \mathrm{sec}$ ); and in the final 32 sessions, there were 10 of each reinforced trial type and 20 of each nonreinforced trial type $($ mean ITI $=90 \mathrm{sec}$ ). Sessions were run 6 days/week.

Next, all subjects received Test 1 , which examined responding during the targets of the "positive"' discrimination in each group: $X$ and $A$ alone and their compounds. In an attempt to examine the effects of the temporal arrangement of the stimuli on performance (rather than on learning), both serial and simultaneous compound presentations were included. There were 12 presentations of each of five trial types: (1) $X \rightarrow 0 \rightarrow 0$, in which a 5-sec presentation of $X$ was followed by two 5 -sec empty periods; (2) $X \rightarrow 0 \rightarrow A$, in which a 5-sec presentation of $X$ was followed, after a 5-sec empty interval, by a 5-sec tone; (3) $X \rightarrow A$, in which the 5-sec presentation of $X$ was followed immediately by a 5 -sec presentation of the tone; (4) $X A$, in which $X$ and the tone were presented simultaneously for $5 \mathrm{sec}$; and (5) $A$, in which the tone alone was presented for $5 \mathrm{sec}$. No reinforcement was available in Test 1 . Trial sequences and ITIs were randomized, as in discrimination training.

All subjects then received another session of discrimination training, identical to the previous ones. This was followed by Test 2 , which tested the negative cues. Test 2 was identical to Test 1 , except that $A$ (the tone) was replaced by $B$ (the noise).

Next, the rats in all groups were given a single session designed to reestablish and maintain responding to the consistently reinforced clicker. Leverpressing was reinforced during each of 605 -sec presentations of the clicker.

Test 3 examined the transfer of $X$ 's modulatory powers by examining its effects on responding evoked by the separately reinforced clicker. Test 3 was identical to Test 2 , except that $B$ (the noise) was replaced by $C$ (the clicker) and responding during clickeralone trials was reinforced.

The next session was designed to extinguish responding to the clicker in all groups; it contained 605 -sec presentations of the clicker, during which sucrose was not available. Finally, Test 4 was designed to test the positive features' abilities to modulate responding to the trained-and-then-extinguished clicker. Test 4 was identical to Test 1 , except that the clicker $(C)$ replaced the tone $(A)$.

Data analysis. We recorded the rate of leverpressing, the percentage of trials on which at least one response occurred, and the latency to the first response, during each 5-sec interval of the CS presentations and the 5-sec pre-CS interval. However, we selected percentage trials with a response as the most appropriate measure, and present only that measure. Response rates did not provide an appropriate measure of discrimination during acquisition, because the rats did not press for several seconds after sucrose was delivered; thus, response rates were artificially depressed during reinforced trials. Neither the latency nor the percentage of trials with a response measure was affected in this manner. Those two measures were very closely related throughout the experiment. Discrimination difference scores were also calculated by subtracting the percentage of nonreinforced trials on which a response occurred from the percentage of reinforced trials on which a response occurred. For the discriminations that involved $A$, these scores were constructed from responding during $A$ on $X A$ trials and on $A$-alone trials; for the discriminations that involved $B$, they were constructed from responding during $B$ on $B$ and $X B$ trials. Although these measures fail to capture the discrimination between $X A$ and $X$ in Group PP and the $X B-X$ discrimination in Group NP, they permit a comparison of the $A-X A$ and $B-X B$ discriminations across the groups of Experiment 1, and with the groups of Experiment 2.

The measure of central tendency reported here is the median. Distribution-free inferential statistics were used throughout, with the level of significance defined as .05 .

\section{Results and Discussion}

There were no reliable within-group differences attributable to the use of the houselight or panel light as $X$. Consequently, that factor is ignored below.

Acquisition. Figure 1 shows leverpressing during the visual feature cue itself and during the 5-sec empty intervals that followed it. In general, control of leverpressing by the feature was consistent with the reinforcement contingencies received by the various groups. The greatest

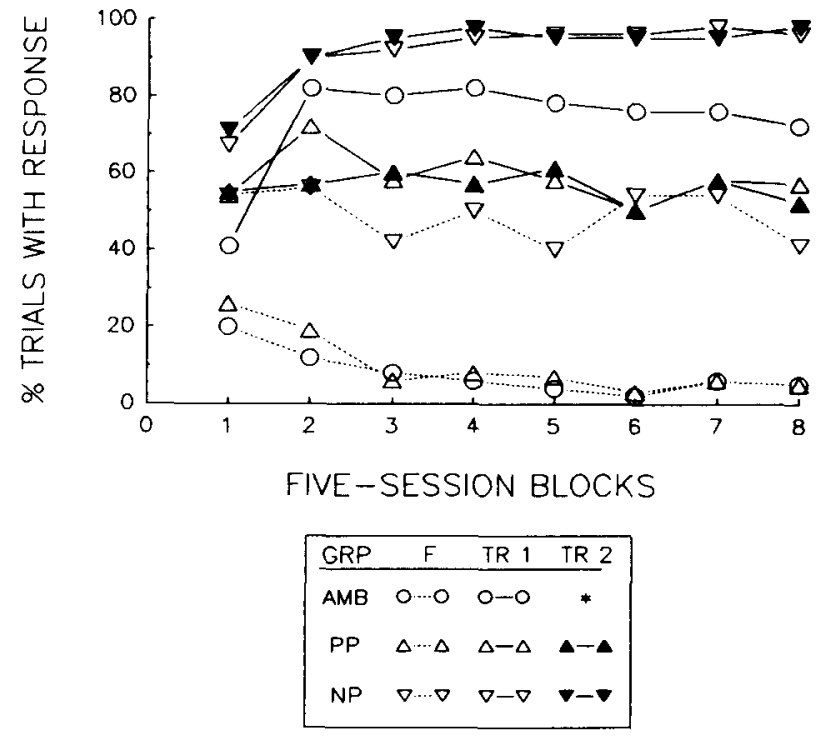

Figure 1. Responding during feature $(X)$ and empty trace intervals in the acquistion phase of Experiment 1. GRP = group, AMB = ambiguous, $\mathbf{P P}=$ positive patterning, $\mathbf{N P}=$ negative patterning, $F=$ responding during the 5-sec visual feature, $T R 1=$ responding during the first 5-sec empty trace interval after the visual feature, TR 2 = responding during the second 5-sec empty trace interval after the visual feature. F and TR 1 responding were collculated on all trials with the feature, and TR 2 responding was calculated on feature-only trials (there were no such trials for Group AMB). 
control over leverpressing was exerted in Group NP, in which $X$ received the full benefit of reinforced trials but shared the costs of nonreinforcement with $B$. The next greatest control was in Group AMB, in which $X$ was both reinforced and nonreinforced in the presence of other cues, and so shared both the benefits of reinforcement and the costs of nonreinforcement with the more contiguous $A$ and $B$ cues. The least responding was observed in Group PP, in which $X$ shared the benefits of reinforcement but bore all the costs of nonreinforcement.

These claims are supported by statistical analyses of the data from the last half of training. Responding during the visual feature itself was reliably greater in Group NP [Mann-Whitney $U(7,7) \leq 5$ ] than in the other two groups, which did not differ from each other. During the empty interval immediately after the feature, however, responding under all three conditions was differentiated. That responding was greater in Group NP than in Group AMB $[U(7,7)=10]$, and greater in Group AMB than in Group PP $[U(7,7)=9.5]$. Similarly, Group NP showed more responding than Group PP during the second 5-sec interval after $X[U(7,7)=3]$.

Figure 2 shows discriminated responding (difference scores) during the target cues over the course of the acquisition phase. The positive-patterning discrimination was easier than the negative-patterning discrimination: the discrimination difference scores were greater in Group PP than in Group NP $[U(7,7)=1]$. Moreover, in Group AMB, the difference scores were greater for the positive discrimination than for the negative discrimination [Wilcoxon

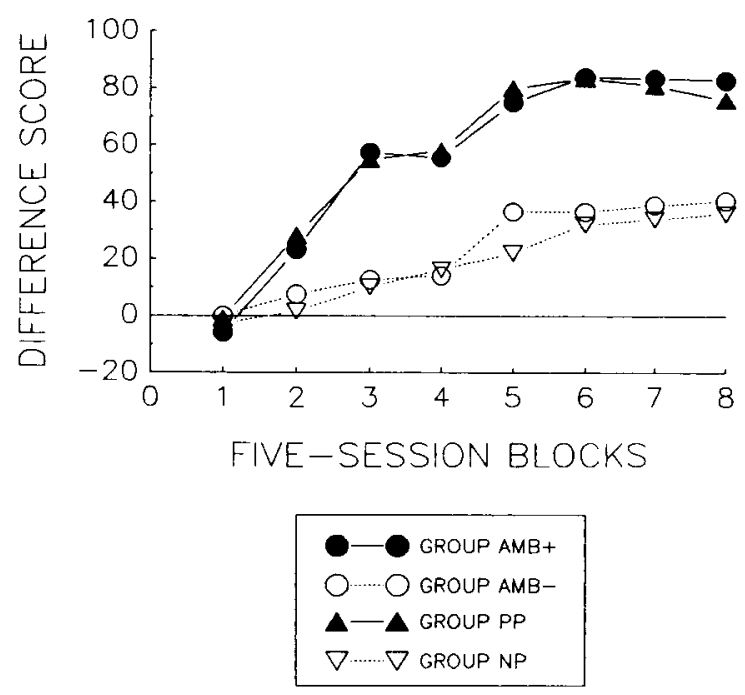

Figure 2. Discriminated responding to the targets in the acquisition phase of Experiment 1. Points represent the percentage of reinforced target trials on which a response occurred minus the percentage of nonreinforced target trials on which a response occurred. $\mathbf{A M B}=$ ambiguous, $\mathbf{P P}=$ positive patterning, $\mathbf{N P}=$ negative patterning. In Group AMB, + signifies performance during the target for which the feature signaled reinforcement $(X \rightarrow A+$ minus $A-)$, and - signifies the target for which the feature signaled nonreinforcement $(B+$ minus $\boldsymbol{X} \rightarrow B-)$.
$T(7)=0$ ]. Because, after the first five-session block, all rats responded during nearly $100 \%$ of the reinforced target trials, these differences primarily reflected differences in responding on nonreinforced trials. (An additional consequence of the high probability of responding on reinforced trials was that the embedded Pavlovian stimulusreinforcer relations were consistent.)

The ambiguous discrimination was no more difficult than the separate patterned discriminations: the rats could use $X$ to signal both the reinforcement of $A$ and the nonreinforcement of $B$ as readily as either function alone. The difference scores for the positive discrimination were no greater in Group AMB than in Group PP $[U(7,7)=23]$, and the difference scores for the negative discrimination were no greater in Group AMB than in Group NP $[U(7,7)$ $=21$ ]. Thus, it is unlikely that $X$ acted simply by modulating the activation threshold for a US representation.

Testing. Figure 3 shows responding during Tests 1-4 in all four groups. Each set of three bars shows responding during the target alone (e.g., $A$ ), during the target on feature $\rightarrow$ empty interval $\rightarrow$ target trials (e.g., $X \rightarrow 0 \rightarrow A$ ), and during the second empty interval after the feature on feature-alone trials (e.g., $X \rightarrow 0 \rightarrow 0$ ). Responding during the targets on serial feature $\rightarrow$ target trials (e.g., $X \rightarrow A$ ) and on simultaneous feature + target trials (e.g., $X A)$ is not shown in Figure 3. In every case, responding on feature $\rightarrow$ empty interval $\rightarrow$ target and feature $\rightarrow$ target trials was similar, and responding on simultaneous compound trials was typically suppressed (relative to responding during other compounds) uniformly across the groups.

Performance on both the $\mathrm{XA}+, A-$ (Test 1) and $B+, X B-$ (Test 2) discriminations was maintained. In Test 1 , responding was more frequent during $A$ on $X \rightarrow 0 \rightarrow A$ trials than during $A$ alone, and more frequent during the second empty interval after $X$ on $X \rightarrow 0 \rightarrow 0$ trials, in both Group PP [Ts $(7)=0]$ and Group AMB $[T \mathrm{~s}(7) \leq 3]$, consistent with the possibility that $X$ acquired the ability to set the occasion for responding to $A$ in those groups. Similarly, in Test 2 , as in training, $X$ suppressed responding to $B$ in Groups AMB and NP; responding to $B$ was lower on $X \rightarrow 0 \rightarrow B$ compound trials than on $B$-alone trials $\left[T_{\mathrm{s}}=0\right]$. At the same time, in Groups AMB and NP, $X$ was strongly excitatory. Moreover, in Groups AMB and NP, the excitatory $B$ might be described as signaling nonreinforcement of $X$ : responding during $B$ on compound trials was reliably lower than responding during the corresponding empty interval on $X$-alone trials in Groups AMB $[T(7)=1]$ and NP $[T(7)=0]$.

Conversely, in Group PP (in which $X$ had been trained as a positive occasion setter), $X$ had no effect on responding to $B[T(7)=13.5]$ and $B$ had no effect on responding in the interval after $X$. Thus, in that group, $X$ 's putative occasion-setting power did not transfer from its original target $(A)$ to the consistently reinforced $B$ target, consistent with the results of both Pavlovian (e.g., Holland, 1983; Rescorla, 1985) and operant (Holland, in press) occasion-setting experiments.

Prior to Test 3, $C$ was retrained as a discriminative stimulus (Sd) for reinforcement of leverpressing in all 


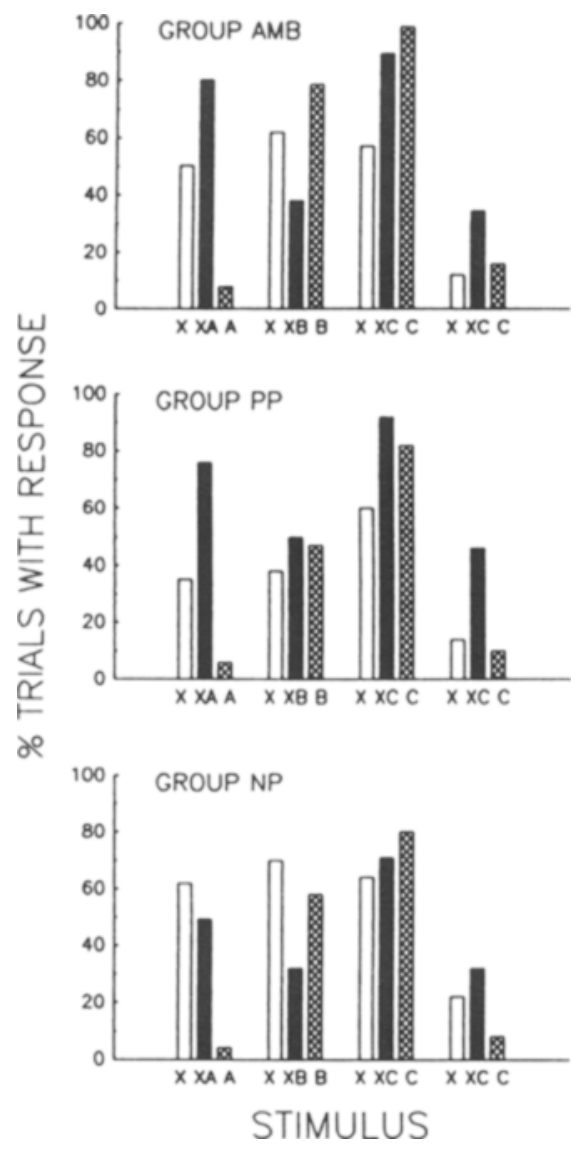

Figure 3. Responding during the four tests in Experiment 1. In each panel, the first trio of bars indicates Test 1 performance, the second trio, Test 2 performance, and so forth. Only responding occurring on test trials with the same temporal sequence as during training is shown. For example, in Test $1, A=$ responding during the 5-sec $A$ target alone, $X A=$ responding during the 5 -sec $A$ target when it was preceded by the 5 -sec $X$ feature and a 5 -sec empty trace interval, and $X=$ responding during the second 5-sec empty trace interval after the $X$ feature on $X$-alone trials. $A M B=$ ambiguous, $\mathbf{P P}=$ positive patterning, $\mathbf{N P}=$ negative patterning.

groups. The third set of bars in each panel of Figure 3 shows Test 3 responding during $C$ on $C$-alone trials, on serial $X \rightarrow 0 \rightarrow C$ trials, and during the corresponding second empty interval on $X$-alone trials. $X$ had no reliable effects on responding controlled by $C$ in any group.

The lack of transfer of $X$ 's suppressive effects to responding evoked by $C$ in Groups NP and AMB is consistent with the findings of Holland (in press, Experiment 2). In that experiment, in which the conditioning preparation, stimuli, and parameters were the same as those used in the present experiment, a serial feature-negative discrimination procedure was used to establish $X$ as a negative occasion setter for responding during $B$. Later, responding was reinforced in the presence of $X$ alone (as in the present negative-patterning procedures). Subsequent tests showed that $X$ maintained its ability to suppress responding during $B$, but did not reduce responding to a separately trained excitor, $C$. It is interesting to speculate that the lack of transfer of $X$ 's suppressive powers to $C$ (in both the present and Holland's, in press, experiment) is related to $C$ 's lack of any of its own inhibitory powers; recall that in Test 2 of the present experiment, in Groups AMB and NP the presence of $B$ (the original excitor) reduced responding that normally occurred in the trace interval after $X$.

The lack of a facilitatory effect of $X$ in Group PP, although consistent with Test 2's data and Holland's (in press, Experiment 1) findings, is uninformative, since responding to $C$ alone was so high that there was little opportunity to observe such an effect.

After Test 3, responding to $C$ was extinguished, and responding to the various stimuli was reassessed in Test 4. The rightmost set of bars in each panel of Figure 2 shows responding in Test 4 . Not surprisingly, there was little evidence that $X$ enhanced responding to $C$ in Group NP. Although responding of those rats was more frequent during $C$ on $X \rightarrow 0 \rightarrow C$ compound trials than on $C$-alone trials $[T(7)=0]$, the frequency was no greater than that in the comparable empty trace interval on $X \rightarrow 0 \rightarrow 0$ trials $[T(7)=13]$, and thus probably reflected only $X$ 's excitatory powers.

Conversely, in both Group PP and Group AMB, $X$ reliably augmented responding to the trained and extinguished $C$. Responding to $C$ on $X \rightarrow 0 \rightarrow C$ trials was more frequent than responding on either $C$-alone trials $[\operatorname{Ts}(7)=0]$ or $X \rightarrow 0 \rightarrow 0$ trials $[T \mathrm{~s}(7)=0]$. Although this transfer is inconsistent with Holland's (1989a) findings with Pavlovian appetitive conditioning procedures with rats, it agrees with Rescorla's (1985) findings with autoshaped keypecking of pigeons and Holland's (in press) observation of transfer to a trained and extinguished cue after serial feature-positive discrimination training with this operant procedure. We have no ready explanation for these discrepancies, although a tempting candidate for speculation may be the differential role of operant response-reinforcer contingencies (explicit or implicit) in those preparations.

$X$ 's action on the trained and extinguished $C$ contrasts with $X$ 's failure to facilitate responding to the consistently reinforced $B$ cue in Test 2 . The selective modulatory ef fect of $\boldsymbol{X}$ on responding to cues that had been both reinforced and nonreinforced has been claimed by some (e.g., Rescorla, 1985) to be characteristic of occasion setters. The simple excitatory strength of $X$ should sum with the strength of any cue, regardless of its training history. Thus, these data suggest that $\boldsymbol{X}$ acted as a positive occasion setter in both Group AMB and Group PP.

Finally, it should be noted that the results of the various test sessions may be complicated by the different overall probabilities of responding across those sessions. For example, responding was greater in Test 3 than in the other tests, probably because of the availability of reinforcement during Test 3 but not the other tests. Furthermore, responding was lower in Test 4 than in the other tests, probably due to the lack of sucrose availability in either Test 4 or the preceding session, in which respond- 
ing to $C$ was extinguished. Experiment 3 examined transfer while a wide range of response levels was maintained.

\section{EXPERIMENT 2}

Experiment 2 was similar to Experiment 1, except that, on compound trials, the elements were presented simultaneously rather than serially. In both Pavlovian and operant procedures with these same cues, Holland (1989a, 1989d, in press) found that the use of simultaneous compounds encouraged rats to solve feature-positive $(X A+, A-)$ and feature-negative $(A+, X A-)$ discriminations by forming simple excitatory and inhibitory associations (respectively) between the feature and the US. However, that strategy would be insufficient to solve $X A+, A-, X-$ discriminations, $X A-, X+, A+$ discriminations, or $X A+, A-$, $X B-, B+$ discriminations.

Many investigators (e.g., Holland, 1989a; Rescorla, 1973) have suggested that under these circumstances, rats abstract cues unique to the stimulus compounds, and independently associate element and compound cues with reinforcement and nonreinforcement, according to the discrimination contingencies. For example, Holland (1989a) showed that with an $X A+, A$ - discrimination, rats associated $X$ and the US, but that with an $X A+, X-, A-$ discrimination, they associated a unique $X A$ compound cue with the US.

To the extent that $X A$ and $X B$ cues were discriminated, then, the $X A+, A-, X B-, B+$ procedure might not be any more difficult than $X A+, X-, A-$ or $X A-, X+, A+$ procedures. Similarly, if the rats learned to respond to particular $X A$ and $X B$ configurations, then transfer of that control to $X C$ compounds would not be expected.

\section{Method}

Subjects and Apparatus. The subjects were 12 male and 12 female albino rats bred in the Psychology Department of Duke University. They were 120 days old at the beginning of the experiment, and had not been involved in previous research. The rats were maintained as in Experiment 1.

All eight of the experimental chambers described in Experiment 1 were used.

Procedure. First, the rats were trained to press the lever, under the control of a clicker, exactly as in Experiment 1.

Next, the rats were divided into three groups of 8 rats each (4 male and 4 female), and discrimination training was begun. The middle part of Table 1 provides an outline of the procedures of Experiment 2. All rats received four kinds of trials in each of the 40 90-min conditioning sessions. The rats in Group AMB received training in which a single visual cue $(X)$ indicated the availability of reinforcement during $A$, and the nonavailability of reinforcement during $B$ : (1) $X A+$ trials comprised a 15 -sec compound of a $1500-\mathrm{Hz}$ ( $74 \mathrm{dBA}$ ) tone and a visual cue; for half of the subjects, the visual cue was the steady illumination of the houselight; for the other half, it was the intermittent $(3 \mathrm{~Hz})$ illumination of the panel light. Leverpresses during the last $5 \mathrm{sec}$ of this compound were reinforced (leverpresses in only the last $5 \mathrm{sec}$ were reinforced in order to make the feature-reinforcement contingencies in this experiment more similar to those of Experiment 1, in which serial compound cues were used). (2) $A$-trials were $15-\mathrm{sec}$ presentations of the tone in which no reinforcement was available. (3) $B+$ trials were 15 -sec presentations of a 73-dBA white noise; leverpresses during the last $5 \mathrm{sec}$ were reinforced. (4) $X B$ - trials comprised a 15 -sec compound of the noise and the visual cue; reinforcement was not available on $X B$ - trials. The rats in Group PP received positive-patterning training, in which reinforcement was available during presentations of a compound of $X$ and $A$, but not during either $X$ or $A$ alone. They received the same kinds of trials as did Group AMB, except that $X B$ - trials were replaced by $X$ - trials, in which the 15 -sec visual cue (houselight for half of the rats and panel light for the others) was presented by itself; reinforcement was unavailable. Finally, the rats in Group NP received negative-patterning training, in which reinforcement was available during presentations of both $B$ and $X$ alone, but not during presentations of the $X B$ compound. They received the same kinds of trials as Group AMB, except that $X A+$ trials were replaced by $\boldsymbol{X}+$ trials, in which reinforcement was available during the last $5 \mathrm{sec}$ of the $15-\mathrm{sec}$ visual cue (houselight for half of the rats and panel light for the others).

Each group received identical trial sequences, which were generated randomly (without constraint) for each session. Intertrial intervals were randomized daily, with the constraint that the range of intervals be from 0.5 to 2.0 times the mean ITI. In the first 4 discrimination training sessions, there were 10 presentations of each trial type (mean ITI $=135 \mathrm{sec}$ ); in the next 4 sessions, there were 15 of each trial type (mean ITI $=90 \mathrm{sec}$ ); and in the final 32 sessions, there were 10 of each reinforced trial type and 20 of each nonreinforced trial type (mean ITI $=90 \mathrm{sec}$ ). Sessions were run 6 days/week.

Next, all subjects received Test 1 , which examined responding during $X, A$, and $B$ alone and during $X A$ and $X B$ compounds in all three groups. No reinforcement was available during any of the stimulus presentations in Test 1 . In Test 1 , all subjects received 12 presentations of each of the five trial types, randomly intermixed.

All subjects then received another session of discrimination training, identical to the previous ones, and a single 90 -min session designed to reestablish and maintain leverpress responding to the clicker. In the latter session, there were 6015 -sec presentations of the clicker, during the last $5 \mathrm{sec}$ in which reinforcement was available. Next, all subjects received Test 2 , which examined responding during $X, B$, and $C$ alone and during $X B$ and $X C$ compounds in all three groups. Test 2 was identical to Test 1 , except that $A$ was replaced by $C$ and reinforcement was available during the last $5 \mathrm{sec}$ of $B$-alone and $C$-alone trials.

Then all subjects were given a single session designed to extinguish responding to the clicker; it consisted of 6015 -sec clicker presentations, during which sucrose was not available. Finally, Test 3 examined responding during $X, A$, and $C$ alone and during $X A$ and $X C$ compounds in all three groups. Test 3 was identical to Test 1 except that $B$ was replaced by $C$.

\section{Results}

The response measures were the same as those used in Experiment 1. However, we present only the percentage of trials on which a response occurred during the final 5 -sec interval of each 15-sec CS. We chose that interval because it was most comparable to responding to the target cues within the serial compound stimuli in Experiment 1.

As in Experiment 1, there were no reliable within-group differences attributable to the use of the houselight or panel light as $X$, so that factor was ignored.

Acquisition. Figure 4 shows the acquisition of the discriminations in the three groups. All subjects eventually mastered their discriminations. Furthermore, performance on neither the positive (involving $X A+$ ) nor the nega- 


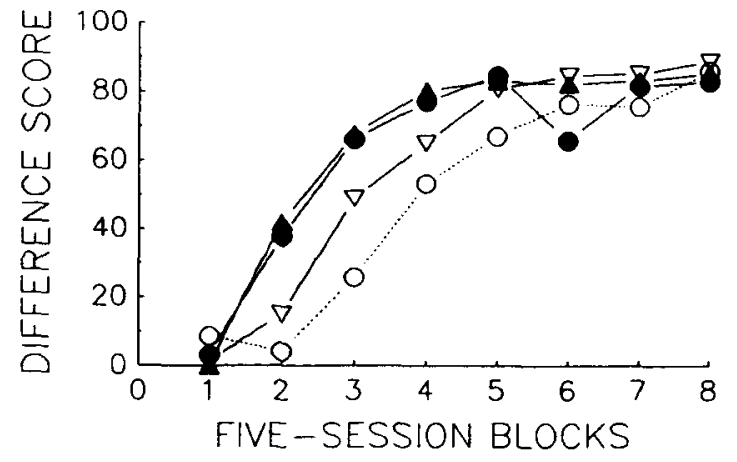

-O GROUP AME+
$O$ O GROUP AME-
$\mathbf{A}-\mathbf{\Delta}$ GROUP PP
$\nabla \cdots . . \nabla$ GROUP NP

Figure 4. Discriminated responding during the targets in the acquisition phase of Experiment 2. Points represent the percentage of reinforced target trials on which a response occurred during the last 5-sec interval minus the percentage of nonreinforced tanget trials on which a response occurred during the last 5-sec interval. $\mathrm{AMB}=$ ambiguous, $\mathbf{P P}=$ positive patterning, $\mathbf{N P}=$ negative patterning. In Group AMB, + signifies performance during the reinforced feature + target compound $(X A+)$ minus responding during $A$ alone, and - signifies performance during the $B$ alone minus responding during the nonreinforeed fenture + target compound $(X B-)$.

tive (involving $X B-$ ) discrimination differed among the groups, both in overall discrimination training sessions and in the first or second halves of training considered separately. This lack of difference was observed with both the difference score measure (shown) and the percentages of reinforced or nonreinforced trials on which a response occurred $[U s(8,8) \geq 21]$. Thus, as in Experiment 1, the presence of $X$ within two compounds with opposite relations with reinforcement had little effect on the ease of compound versus element discrimination.

Also as in Experiment 1, in Group AMB, the positive discrimination involving $X \boldsymbol{A}+$ was learned more rapidly than the negative discrimination involving $X B-$ : the $B-X B$ difference scores were reliably greater than the $X A-A$ difference scores during the first half of training $[T(8)=0]$ but not during the second half $[T(8)=12]$. However, unlike in the previous experiment, discrimination performance of Groups PP and NP did not differ reliably over either the first or second half of training $[U s(8,8) \geq 18]$.

Testing. The left portions of the panels of Figure 5 show responding during Test 1 . In all groups, the original discriminations were maintained $[T \mathrm{~s}(8)=0]$; as at the end of training, there were no differences in the discrimination difference scores among the groups that had been trained with each discrimination $[U \mathrm{~s}(8,8) \geq 19.5]$. Not surprisingly, however, Group PP showed no evidence of a $B, X B$ discrimination $[T \mathrm{~s}(8)=16]$. Nor did Group NP respond more to $X A$ than to $A[T(6)=10]$; indeed, in that group, responding was less during $X A$ than during $X$ alone, suggesting that $A$ had inhibitory properties. Although it might be argued that the suppression of responding during $X A$ was the consequence of generalization between the $X A$ and $X B$ compounds, several aspects of these data argue against that conclusion. First, in Test 1 , excellent discriminations between $A$ and $B$ and between $X$ and the $X A / X B$ compounds were evident. Second, later, in Test 2, the rats in Group NP showed excellent discrimination between the $X B$ and $X C$ compounds, which in simple conditioning experiments in our laboratory have been seen to be as similar as the $X B$ and $X A$ compounds.

$X$ alone was excitatory in Group NP but not in Groups AMB and PP; responding to $X$ was greater in Group NP than in either of the latter two groups $\left[U_{s}(8,8) \leq 11\right]$. Thus, because responding during the compounds does not reflect a simple summation of responding to the elements,

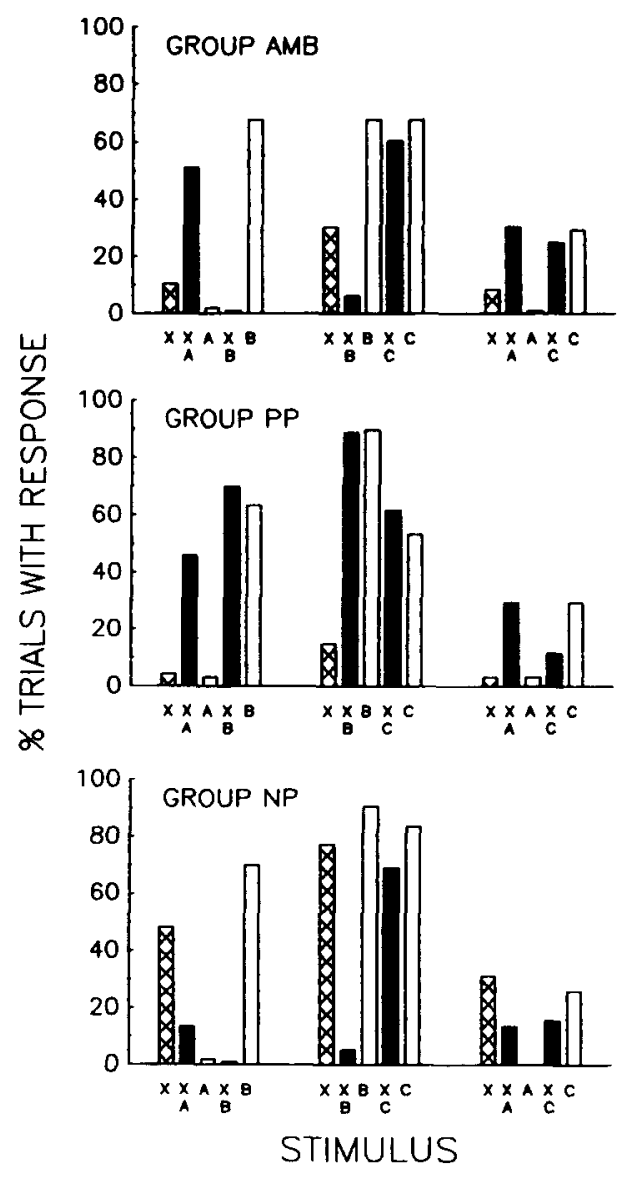

Figure 5. Responding during the three tests in Experiment 2. In each panel, the first set of bars indicates Test 1 performance, the second set, Test 2 performance, and the third, Test 3 performance. Each bar indicates the percentage trinls on which a response cocurred during the final 5-sec interval of each cue. For example, in Test 1 , $A=$ responding during the last 5-sec interval of the $A$ target alone, $\mathrm{XA}=$ responding during the last 5-sec of the $\mathrm{XA}$ compound, and so forth. $\mathbf{A M B}=$ ambiguous, $\mathbf{P P}=$ positive patterning, $\mathrm{NP}=$ negative patterning. 
the results of Test 1 implicate configural processes (or occasion setting) in the solution of the $X A+, A$ - discrimination in Groups AMB and PP and in the solution of the $B+, X B$ - discrimination in Group NP.

The center portion of each of the panels of Figure 5 shows responding during Test 2 . As in Test 1 , the discriminations that involved $B$ were maintained in each group that had received that training. However, $X$ had no reliable effect on responding to $C$ in any of the groups $[T \mathrm{~s}(8) \geq 14]$, again consistent with a configural process (or occasion setting).

The right portion of each of the panels of Figure 5 shows responding during Test 3 after the extinction of responding to $C$. The discriminations that involved $A$ were maintained in Groups AMB and PP [Ts $(8)=0]$, albeit at a lower level than in Test 1 . Once again consistent with a configural notion, $X$ 's ability to augment responding to $A$ did not transfer to $C$ in any group $[T \mathrm{~s}(8) \geq 14]$. However, because the lack of reinforcer availability in Test 3 and the preceding extinction session apparently had a general decremental effect on responding, Test 3 may not have provided a sensitive test of transfer. Experiment 3 examined transfer while responding to the transfer targets was maintained at a variety of levels.

\section{EXPERIMENT 3}

Experiment 3 further examined performance on the ambiguous $X A+, A-, B+, X B-$ discrimination with serial and simultaneous compounds. It extended the results of Experiments 1 and 2 in several ways. First, the simultaneous and serial procedures were compared within the same experiment, with more closely analogous test procedures. Second, performance with serial 5-sec feature $\rightarrow$ 5 -sec trace $\rightarrow 5$-sec target compounds was compared with performance with simultaneous compounds of both 15$\mathrm{sec}$ (equating feature-reinforcement interval) and 5-sec (equating target-reinforcement interval) durations. Third, possible confounds of generalization between the originaland transfer target cues were reduced by using target cues of different modalities.

Fourth, Experiment 3 used more sensitive tests of transfer to target cues of a variety of different training histories: consistently reinforced, consistently nonreinforced, reinforced and then nonreinforced, and nonreinforced and then reinforced. To accomplish this aim, after training with the ambiguous $X A+, A-, B+, X B-$ discrimination, a simple discrimination was trained between two transfer target cues $(C+, D-)$. After a transfer test, the target discrimination was reversed $(C-, D+)$ and additional transfer tests were conducted as that discrimination underwent reversal. Because of this interspersion of testing and reversal training, transfer could be assessed at many levels of responding during various kinds of target stimuli.

\section{Methods}

Subjects and Apparatus. The subjects were 24 male SpragueDawley-strain rats obtained from Charles River Laboratories,
Raleigh, NC. At the beginning of the experiment, they were 165 days old and experimentally naive. They were maintained as in Experiments 1 and 2 .

The apparatus was the same as that used in Experiment 2, except that during the first eight sessions, $2 \times 4 \mathrm{~cm}$ paper clamps were attached to each lever to aid in leverpress training.

Procedure. First, the rats were trained to consume sucrose from the food cup and to press the levers. In each of four 60 -min sessions, the rats received a $0.3-\mathrm{ml}$ delivery of $0.2 \mathrm{M}$ sucrose for each leverpress. In addition, during the first $40 \mathrm{~min}$ of the first two sessions, sucrose deliveries occurred on a VT 2 -min schedule.

Next, the rats were assigned to three groups of 8 rats each, and given reinforced training with each of the cues that were reinforced in the subsequent discrimination phase (this procedure ensured that the rats responded on all scheduled reinforced trials when discrimination training procedures were introduced). In each of five sessions, all rats received 30 presentations each of an $8-\mathrm{Hz}, 70-\mathrm{dB}$ clicker, $B$, and a compound cue comprising two other auditory cues, $X$ and $A$. For half of the rats in each group, $X$ was a $1500-\mathrm{Hz}$ tone ( $72 \mathrm{~dB}$ ) and $A$ was a $72-\mathrm{dB}$ white noise; for the other half, the identities of $X$ and $A$ were reversed. The trials were randomly intermixed within each session. In Group Ser, $B$ was $5 \mathrm{sec}$ in duration and the compound consisted of a 5-sec presentation of $X$ followed, after a 5-sec empty trace interval, by a 5-sec presentation of $A$. Sucrose was delivered for each leverpress during $A$ and $B$. In Group $\mathrm{Sim}-5, B$ was $5 \mathrm{sec}$ in duration and the compound was a 5 -sec simultaneous compound of $X$ and $A$; sucrose was continuously available during $B$ and this compound. In Group Sim-15, $B$ was $15 \mathrm{sec}$ in duration and the compound was a 15-sec simultaneous compound of $X$ and $A$; sucrose was available during the last $5 \mathrm{sec}$ of the $B$ and $X A$ compound presentations. Sessions $1-3$ were $30 \mathrm{~min}$ in duration; Sessions $4-5$ of this phase and all subsequent sessions in this experiment were 90 min long.

Next, the rats received training on the ambiguous $X A+, A-, B+$, $X B$ - discrimination. Table 2 gives an outline of these and the remaining procedures of Experiment 3 . The rats in all three groups received continued training with reinforced $B+$ and $X A+$ trials like the ones they had received in the conditioning phase. Intermixed with those trials, the rats in Group Ser received nonreinforced 5-sec $A$-alone presentations and nonreinforced serial compounds that consisted of a 5-sec presentation of $X$ followed, after a 5-sec empty trace interval, by a 5-sec presentation of $B$. The rats in Group Sim-5 received nonreinforced 5-sec presentations of $A$ and nonreinforced presentations of an $\boldsymbol{X B}$ compound intermixed with their original training trials. The rats in Group Sim-15 received nonreinforced 15 -sec $A$ presentations and nonreinforced 15 -sec presentations of the $X B$ compound.

In Sessions 1-16 of the discrimination training phase, there were 20 of each type of trial in each session. In the remaining sessions of this phase, there were 10 of each of the two types of reinforced trials and $\mathbf{3 0}$ of each of the two kinds of nonreinforced trials. In an effort to equate the terminal discrimination performance of the three groups, different numbers of these additional sessions were administered to each group of rats. Group Ser received 24 additional sessions ( 40 sessions total), Group Sim-5 received 14 additional sessions ( 30 sessions total), and Group Sim-15 received 4 additional sessions (20 sessions total).

After finishing the discrimination training phase, each group was given training with the cues to be used as targets in the transfer tests. This training was designed to establish responding to one visual cue (V1) but not another (V2). $V 1$ and $V 2$ were an intermittent $(3 \mathrm{~Hz})$ illumination of the houselight and a steady illumination of the panel light, counterbalanced within each group. $V 1$ and $V 2$ were $5 \mathrm{sec}$ long in Groups Ser and Sim-5 and 15 sec long in Group Sim-15.

In the first two sessions of this phase, subjects in all groups received 40 presentations of $V 2$, during which sucrose was unavailable $(V 2-)$, and 40 presentations of $V 1$, during which sucrose was available for each leverpress $(V 1+)$. In addition, in an attempt to 
Table 2

Outline of Procedure of Experiment 3

\begin{tabular}{|c|c|c|c|c|c|}
\hline & Discrimination & $V 1+, V 2-$ & Test 1 & $V 1-, V 2+$ & Tests 2-6 \\
\hline Group SER & $\begin{array}{l}X \rightarrow 0 \rightarrow A+, A- \\
X \rightarrow 0 \rightarrow B-, B+\end{array}$ & $V 1+, V 2-$ & $\begin{array}{l}X \rightarrow 0 \rightarrow V 1-, V 1- \\
X \rightarrow 0 \rightarrow V 2-, V 2- \\
X \rightarrow 0 \rightarrow A-, A- \\
X \rightarrow 0 \rightarrow B-, B-, X- \\
X \rightarrow 0 \rightarrow A+, V 1+\end{array}$ & $V 1-, V 2+$ & $\begin{array}{l}X \rightarrow 0 \rightarrow V 1-, V 1- \\
X \rightarrow 0 \rightarrow V 2-, V 2- \\
X \rightarrow 0 \rightarrow A-, A- \\
X \rightarrow 0 \rightarrow B-, B-, X- \\
X \rightarrow 0 \rightarrow A+, V 2+\end{array}$ \\
\hline $\begin{array}{l}\text { Group SIM-5 } \\
\text { and } \\
\text { Group SIM-15 }\end{array}$ & $\begin{array}{l}X A+, A- \\
X B-, B+\end{array}$ & $V 1+, V 2-$ & $\begin{array}{l}X V 1-, V 1- \\
X V 2-, V 2- \\
X A-, A- \\
X B-, B-, X- \\
X A+, V 1+\end{array}$ & $V 1-, V 2+$ & $\begin{array}{l}X V 1-, V 1- \\
X V 2-, V 2- \\
X A-, A- \\
X B-, B-, X- \\
X A+, V 2+\end{array}$ \\
\hline
\end{tabular}

Note- + signifies availability of reinforcement, - signifies no reinforcement available, $B$ was a clicker, $X$ and $C$ were a tone and a noise, counterbalanced, $V 1$ and $V 2$ were a houselight and a panel light, counterbalanced. In Groups SER and SIM-5, all stimuli were $5 \mathrm{sec}$ in duration; in Group SIM-15, they were $15 \mathrm{sec}$ long. A V1,V2 training session occurred before each test session.

encourage initial acquisition of responding during $V 1, V 1$ presentations were followed immediately by a response-independent sucrose presentation (see, e.g., Peterson, Ackil, Frommer, \& Hearst, 1972). However, because the novel visual cues produced considerable disruption, some of the subjects failed to acquire leverpress responding during this procedure. Consequently, the rats were returned to their original discrimination training contingencies for a single session, and then, in the next session, the $V 1+$ and $V 2-$ trials were reintroduced, intermixed with the original discrimination trials. That session comprised seven presentations of each of the 4 types of original discrimination trials, $14 \mathrm{~V} 2-$ trials, and $14 V 1+$ trials; response-independent sucrose was also delivered immediately after each $V 1+$ trial. Next, all subjects received one more $V 1+, V 2$ - discrimination training session, identical to the first two sessions of this phase, except that no response-independent sucrose was delivered.

All groups then received transfer testing. This phase included six test sessions, each separated by a reversal training session in which the reinforcement contingencies during $V 1$ and $V 2$ were transposed. In each test and reversal training session, all subjects received cues of the same durations and temporal arrangement that they had received in discrimination and transfer target training.

Test Session 1 examined transfer of $X$ 's powers to the consistently reinforced $V_{1}+$ and the consistently nonreinforced $V 2-$. There were seven presentations of each of 12 types of trials, randomly intermixed. Six of those trial types, $X A+, A-, X B-, B+$, $V 1+, V 2-$, were identical to those received in the previous training. Two were nonreinforced transfer test trials, which examined the effects of the ambiguous $X$ feature on responding to the consistently reinforced $V 1(X V 1-)$ and the consistently nonreinforced $V 2(X V 2-) . X$ - trials examined responding during $X$ alone (and, in Group Ser, during two empty trace intervals after $X$ ). Finally, there were nonreinforced presentations of the three cues that were normally reinforced $(X A-, B-$, and $V 1-)$. These trials were included in order to make possible the use of response rate measures (see the end of the Procedure section of Experiment 1), which were not reported here.

Next, a single session started the reversal training of $V 1$ and $V 2$. In that session, there were 20 nonreinforced presentations of $V 1-$ and 20 presentations of $V 2+$, during which each leverpress was followed by sucrose delivery; response-independent sucrose was delivered immediately after each $V 2+$ presentation. In addition, intermixed with those trials were $5 X A+, 15 A-, 5 B+$, and 15 $X \boldsymbol{B}$ - presentations.

Test Sessions 2-6 examined transfer of $X$ 's powers to $V 1$ while that cue underwent extinction and to $V 2$ while that cue acquired control of leverpressing. Test Session 2 was the same as Test 1 , except that the reinforcement contingencies during $V 1$ and $V 2$ were reversed and additional nonreinforced $V 2$ trials, rather than $V 1$ trials, were given. Next, another reversal training session was given; this session was identical to the previous one, except that there were $10 V 1-$ and $30 V 2+$ trials. Finally, Test Sessions 3-6 were identical to Test Session 2 and were interspersed with additional reversal training sessions, identical to the second one.

\section{Results and Discussion}

Acquisition. The acquisition of the ambiguous discrimination proceeded uneventfully. The discrimination was learned most rapidly by Group Sim-15 (asymptotic after 20 sessions), next fastest by Group Sim-5, asymptotic after 30 sessions), and most slowly by Group Ser. Terminal performance was successfully matched by Groups Sim-5 and Sim-15, but Group Ser never achieved the accuracy of those groups, as can be seen from the left panels of Figure 6, which show performance on the original discrimination during the transfer test sessions. In fact, the performance of Group Ser was obviously poorer than that of Group AMB of Experiment 1 (cf. Figure 2). A likely explanation for this difference is the use of feature and target cues of the same modality in Experiment 3 but of different modalities in Experiment 1. Although the present experiments do not permit appropriate comparisons, Holland (1989a) found that the use of unimodal stimuli slowed the acquisition of serial (but not of simultaneous) Pavlovian appetitive feature-positive and feature-negative discriminations.

Two rats in each of the three groups failed to acquire leverpressing during the appropriate visual cues in the transfer target training phase.

Transfer tests. Figure 6 shows the test performance of the remaining 6 rats in each group. All subjects maintained their original discrimination performance (left panels): $X$ retained its ability to concomitantly enhance responding to $A$ and suppress responding to $B$. All subjects responded more on $X A+$ and $B+$ trials than on $A-$ and $X B$ - trials $[T \mathrm{~s}(6)=0$ ], but the discrimination difference scores were significantly smaller $[U s(6,6) \leq 6]$ and responding on nonreinforced trials was reliably greater $[U s(6,6) \leq 8.5]$ in Group Ser than in the other two groups.

Nevertheless, $X$ showed little evidence of modulating performance to $V 1$ or $V 2$ in any group, except for the trained-and-extinguished $V 1$ after reversal training in 

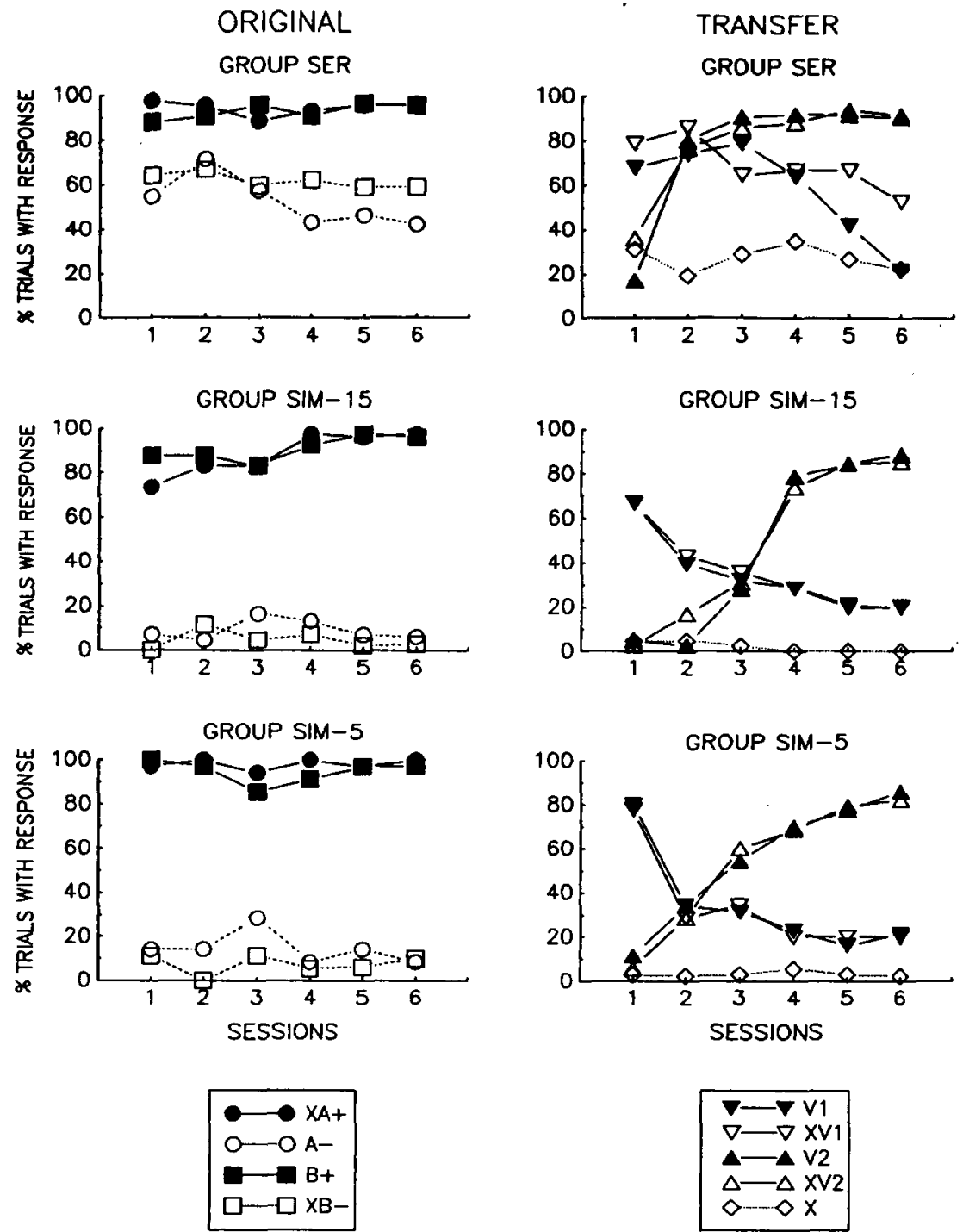

Figure 6. Responding during the six transfer test sessions of Experiment 3. The left panels show responding during the original training cues in those sessions, and the right panels show responding on the transfer test trials in those sessions. $V 1$ was reinforced and $V 2$ nonreinforced prior to Test Session 1; $V 1$ was nonreinforced and $V 2$ reinforced in sessions prior to each of the subsequent transfer test sessions. Thus, in Test Session 1, V1 was a consistently reinforced cue and $V 2$ was a consistently nonreinforced cue, and in subsequent test sessions, $V 1$ was a trained and extinguished cue and $V 2$ was a consistently reinforced cue.

Group Ser (right panels of Figure 6). In Groups Sim-5 and Sim-15 there were no reliable differences in responding during $X V 1$ and $V 1$ or between $X V 2$ and $V 2$ during any test session $[T \mathrm{~s}(6) \geq 7.5]$. In Group Ser, $X$ did not suppress responding to $V 1$ while $V 1$ evoked substantial responding, but did facilitate responding to $V 1$ after $V 1$ was extinguished [Sessions 5 and 6, $T(6)=2$ ]. Furthermore, $X$ did not enhance or suppress responding to $V 2$ in any session of $V 2$ 's training [ $T \mathrm{~s}(6) \geq 6.5$ ], except in Session 1 . In that session, there was marginally more responding during $X V 2$ than during $V 2$ alone $[T(6)=3.5]$.
However, that superiority probably does not reflect transfer of $X$ 's occasion-setting powers, because responding was not reliably greater during $V 2$ after $X$ than during the corresponding empty interval after $X$ alone $[T(6)=10.5]$.

\section{GENERAL DISCUSSION}

Ambiguous $X A+, A-, X B-, B+$ discriminations in which $X$ indicated when responding during one cue would be reinforced and when responding during another cue would not be reinforced were no more difficult than dis- 
criminations in which $X$ signaled only the availability or nonavailability of reinforcement during another cue. It is unlikely that the solution of these ambiguous discriminations involved $X$ 's modulation of the activation threshold of a US representation (e.g., Rescorla, 1985): $X$ might raise or lower that threshold, but to raise it for $B$ and lower it for $\boldsymbol{A}$ requires more specificity of $X$ 's action than admitted by Rescorla's proposal.

Several accounts consistent with that specificity can be distinguished. First, Rescorla's (1985) view might be preserved if it were assumed that in the ambiguous discriminations $X$ acquired only one type of modulatory power but also the complementary simple associative strength. For example, $X$ may have raised the activation threshold of the US representation (negative occasion setting) and also directly activated that representation (excitation). Alternatively, $X$ may have served both as a positive occasion setter and as a conditioned inhibitor. As long as simple associative and modulatory powers were acquired relatively independently (as Rescorla suggested), either of these strategies could be used to solve the ambiguous discriminations with no more difficulty than the patterned discriminations. However, the absence of substantial transfer to other targets argues against these options; Holland (in press) found substantial transfer of simple excitation and inhibition with these discrete-trial operant procedures.

A second account is Holland's (1983) claim that occasion setters' action is specific to stimulus-reinforcer pairs. In that case, there is no conflict between $X$ 's facilitating the action of an $A$-reinforcer association and inhibiting the action of a $B$-reinforcer association. Thus, the acquisition of the ambiguous discrimination should be no more difficult than the acquisition of the patterned discriminations, and little transfer to new targets would be anticipated.

A third account denies the involvement of any special modulatory processes. Within this "configural" approach (e.g., Wilson \& Pearce, 1989), the four types of trials are viewed as four separate stimuli, each with its own relation to reinforcement. That is, $X$ does not serve as a modulator, but simply contributes to the identity of the cues $X A$ and $X B$. The rats respond differentially to those two cues and to separate $A, B$, and $X$ presentations simply because they are different cues with separate contingencies of reinforcement. As long as $X A$ and $X B$ do not generalize significantly to each other, there would be little reason to expect learning of the ambiguous discrimination to be more difficult than learning that involved only one of those cues. Similarly, unless there were significant generalization between $X C$ and $X A$ and/or $X B$, little transfer to the $C$ targets would be anticipated.

The present data provide little evidence to distinguish these latter alternatives. Although Holland (1989a) provided evidence that in simple Pavlovian positive-patterning $(X A+, A-, X-)$ discriminations, the use of serial compounds encouraged the use of modulatory, occasionsetting strategies and the use of simultaneous compounds encouraged configural processes, it is possible that a single mechanism was responsible for the solution of both the simultaneous and serial discriminations in the present experiments. For example, Holland (1989c) and Rescorla (1989) showed that modulatory (occasion setting) processes can emerge with simultaneous compounds under some circumstances. Similarly, in principle, it is not unreasonable that rats would configure the elements of both serial and simultaneous compounds.

Although the patterns of data found with serial and simultaneous cues were, on the whole, similar in the present experiments, one finding suggests that the serial and simultaneous discriminations might have been solved with different strategies. Although responding during a trained and extinguished cue was unaffected by a simultaneously trained $X$ in Experiments 2 and 3, responding during such a target was facilitated by a serially trained $X$ in Experiments 1 and 3. This differential transfer is consistent with previous findings from serial feature-positive discriminations in this conditioning preparation (Holland, in press). The further observation that after serial training $X$ did not augment responding in the presence of consistently reinforced or nonreinforced stimuli makes it unlikely that $X$ 's facilitation of responding during trained and extinguished targets in the present experiments was due solely to excitation controlled by $X$ or to generalization between $X \rightarrow C$ and $X \rightarrow A$ trials.

Unfortunately, in the present experiments, it is not certain whether this differential transfer was the consequence of the temporal arrangements of the cues during training or at the time of testing. In Experiments 2 and 3, training and test compounds were always of the same temporal arrangement, and in Experiment 1, after training with serial compounds, simultaneous test compounds yielded no information: there was very little responding to any such compound, including ones that evoked considerable responding when the elements were presented serially. However, previous data from Pavlovian procedures showed transfer to be determined by the temporal arrangement of cues during training rather than at the time of testing.

Nevertheless, it must be noted that the transfer of $X$ 's modulatory powers to the trained and extinguished cue observed here and in Holland's (in press) experiments is inconsistent with Holland's (1983) view that occasion setters act on specific CS-US associations. If occasion setters act specifically, then transfer to other target cues (apart from generalization) should not occur, regardless of the training histories of those targets. Apparently, in this preparation, as in pigeon autoshaping (Rescorla, 1985), the range of transfer targets for occasion setters is broader, including other types of cues that have been both reinforced and nonreinforced. That range is not complete, however: both Rescorla (1985) and we (the present experiments) found no evidence of transfer to consistently reinforced or untrained cues.

The data that we report here are also inconsistent with a more recent version of Holland's (1983) view of occasion setting. Holland $(1989 a, 1989 d)$ found that a cue trained as an occasion setter with one target cue modulated responding controlled by a target of another occa- 
sion setter. At the same time, that occasion setter did not modulate responding controlled by cues that were not trained as targets of occasion setters (e.g., cues that were consistently reinforced). Those transfer data are clearly incompatible with Holland's (1983) notion, and they led Holland (1989a, 1989d) to propose a multiple memory systems account of occasion setting. Within that view, stimuli involved in occasion-setting discriminations are represented both in a memory system involved in simple association and in a higher level system; stimuli involved only in simple associative relations (and not occasion setting) are represented only in the former system. Furthermore, transfer was assumed to occur readily only among cues represented within a memory system. Thus, only stimuli that were themselves trained as targets of occasion setters would be suitable targets of transfer from other occasion setters.

For the transfer data of the present experiments to be compatible with that view, it must be assumed that stimuli may be represented in the higher level memory system after a broader range of training procedures than Holland (1989a, 1989d) suggested. Furthermore, the independence of the positive and negative occasion-setting functions of $X$ observed here would require that within the higher level system transfer be at least somewhat limited: targets of negative occasion setters could not be suitable targets of positive occasions setters, and vice versa. Further exploration of the conditions for transfer are necessary in order to determine if Holland's (1989a, 1989d) view maintains any heuristic value.

Finally, some comment on the relationship of the present experiments to those that have investigated occasion setting with Pavlovian contingencies alone is in order. It might be argued that even a simple operant discrimination involves stimulus control akin to occasion setting, because the Sd sets the occasion for responding based on the response-reinforcer relation. A simple operant Sd may be functionally like a feature in a Pavlovian positivepatterning $(X \rightarrow A+, A-, X-)$ discrimination: responses $(A)$ are reinforced $(+)$ in the presence of the $S d(X)$, but reinforcement is not delivered in the presence of $X$ unless $A$ occurs, and $A$ is not reinforced unless $X$ is present.

Indeed, recent data suggest that Pavlovian occasion setters have much in common with operant discriminative stimuli. For example, Davidson, Aparicio, and Rescorla (1988) found that a cue trained as an operant Sd effectively set the occasion for responding to a cue that had been trained as a target in Pavlovian occasion setting, and a Pavlovian occasion setter effectively controlled an operant that was trained with a different Sd.

Consequently, within operant discrimination procedures, the use of interstimulus relations known to generate Pavlovian occasion setting might be analogous to arranging "higher order" occasion-setting relations in Pavlovian conditioning procedures (e.g., Arnold, Grahame, \&
Miller, 1991; Sidman, 1986). For example, in the experiments reported here, $X$ might be said to indicate when $A$ signals the viability of the leverpress $\rightarrow$ sucrose relation. At present, we have no evidence for whether our findings depend solely on the Pavlovian contingencies embedded in our procedures, the explicit operant contingencies, or some combination of those contingencies. Thus, it would be of considerable interest to further compare the properties of operant conditional discrimination performance with those of both simple and "higher order" Pavlovian conditional discrimination performance.

\section{REFERENCES}

Arnold, H. M., Grahame, N. J., \& Miller, R. R. (1991). Higherorder occasion setting. Animal Learning \& Behavior, 19, 58-64.

Davidson, T. L., Aparicio, J., \&escorla, R. A. (1988). Transfer between Pavlovian facilitators and instrumental discriminative stimuli. Animal Learning \& Behavior, 16, 285-291.

Holland, P. C. (1983). Occasion-setting in Pavlovian feature positive discriminations. In M. L. Commons, R. J. Herrnstein, \& A. R. Wagner (Eds.), Quantitative analyses of behavior: Discrimination processes (Vol. 4, pp. 183-206). New York: Ballinger.

Holland, P. C. (1989a). Acquisition and transfer of conditional discrimination performance. Joumal of Experimental Psychology: Animal Behavior Processes, 15, 154-165.

Holland, P. C. (1989b). Feature extinction enhances transfer of occasion setting. Animal Learning \& Behavior, 17, 269-279.

Holland, P. C. (1989c). Occasion setting with simultaneous compounds in rats. Journal of Experimental Psychology: Animal Behavior Processes, 15, 183-193.

Holland, P. C. (1989d). Transfer of negative occasion setting and conditioned inhibition across conditioned and unconditioned stimuli. Journal of Experimental Psychology: Animal Behavior Processes, 15, 311-328.

Holland, P. C. (in press). Acquisition and transfer of occasion setting in operant feature positive and feature negative discriminations. Learning \& Motivation.

MoOre, J. W., Newman, F. L., \& Glasgow, B. (1969). Intertrial cues as discriminative stimuli in human eyelid conditioning. Journal of Experimental Psychology, 79, 319-326.

Peterson, G. B., ACkil, J. E., Frommer, G. P., \& Hearst, E. (1972). Conditioned approach and contact behavior towards signals for food or brain-stimulation reinforcements. Science, 177, 1009-1011.

Rescorla, R. A. (1973). Evidence for a "unique stimulus" account of configural conditioning. Joumal of Comparative \& Physiological Psychology, 85, 331-338.

RESCORLA, R. A. (1985). Conditioned inhibition and facilitation. In R. R. Miller \& N. E. Spear (Eds.) Information processing in animals: Conditioned inhibition (pp. 299-326). Hillsdale, NJ: Erlbaum.

RESCORLA, R. A. (1989). Simultaneous and sequential conditioned inhibition in autoshaping. Quarterly Journal of Experimental Psychology, 41B, 275-286.

Sidman, M. (1986). Functional analysis of emergent verbal classes. In T. Thompson \& M. D. Zeiler (Eds.), Analysis and integration of behavioral units. Hillsdale, NJ: Erlbaum.

SKINNER, B. F. (1938). The behavior of organisms. New York: Appleton-Century.

Wilson, P. N., \& Pearce, J. M. (1989). A role for stimulus generalization in conditional discrimination learning. Quarterly Journal of Experimental Psychology, 41B, 243-273.

(Manuscript received June 6, 1990; revision accepted for publication January 20, 1991.) 